\section{Subcutaneous furosemide in advanced heart failure: has clinical practice run ahead of the evidence base?}

\author{
James M Beattie, ${ }^{1}$ Miriam J Johnson²
}

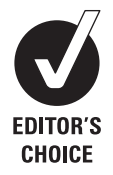

Over the past 20 years, progress in our understanding of the nature and treatment of congestive heart failure due to left ventricular systolic dysfunction has significantly improved the outlook of those diagnosed with this condition and many patients now live longer and more comfortable lives. Comprehensive heart failure care is largely founded on the results of robust randomised clinical trials of drug, device and cardiac surgical interventions which are enshrined as the basis of widely implemented clinical guidelines. Current heart failure treatment may thus be regarded as an example of the application of evidence based medicine.

Despite such developments, heart failure remains burdensome for patients and their families, and is often complicated by prognostic uncertainty and comorbidities common in the typically affected older population. Heart failure still tends to progress inexorably and it is now acknowledged that those with palliative care skills should be central to the multidisciplinary team approach to this ultimately fatal illness. ${ }^{1}$ Their contribution is predominantly relevant to those with advanced disease or approaching the end of life. Indeed such heart failure patients are increasingly treated by specialist palliative care teams in hospitals, hospices and in the community.

Provision of therapy via the subcutaneous (SC) route is common in palliative care practice for the continuous

1Department of Cardiology, Heart of England NHS Foundation Trust, Birmingham, UK; National Clinical Lead, NHS Heart Improvement.

${ }^{2}$ Department of Health Sciences, Hull York Medical School, University of Hull; St Catherine's Hospice, Scarborough, UK

Correspondence to Dr James M Beattie, Department of Cardiology, Heart of England NHS Foundation Trust, Heartlands Hospital, Bordesley Green East, Birmingham B9 5SS, UK;

james.beattie@heartofengland.nhs.uk administration of medication for symptom relief. However, with the exception of opioids such as morphine and hydromorphone, few drugs have randomised controlled data comparing SC and intravenous routes of administration. Many drugs are thus used outside their regulatory license in terms of clinical indication and mode of delivery. This is acceptable when the prescriber can demonstrate that their practice accords with a consensus based on an appropriate body of professional experience and opinion. There are also guidelines regarding the use of drugs off-license which highlight the importance of an appropriate discussion of such a management plan with the patient. ${ }^{2}$

Over the past decade, the use of SC furosemide to treat congestion in endstage heart failure has increased, particularly in the field of palliative care. There is clearly a growing body of professional opinion, but is this treatment protocol supported by the parallel development of an evidence base? Evidence based medicine has been defined as 'the integration of best research evidence with clinical expertise and patient values'. ${ }^{3}$ We shall consider the use of SC furosemide for advanced heart failure in terms of these three domains.

\section{BEST RESEARCH EVIDENCE}

Furosemide is unlicensed for SC use. However, the diuretic effect of this mode of administration has been demonstrated in a randomised placebo controlled trial of 12 healthy normal volunteers aged 22-56 years (mean 36), when the SC bolus injection of $20 \mathrm{mg}$ of furosemide elicited a statistically significant diuretic and natriuretic response lasting up to 4 h. ${ }^{4}$ A subsequent report confirmed the clinical effectiveness of this route of drug delivery in the treatment of 8 'home hospice' advanced heart failure patients aged 58-79 years (mean 72) with congestion resistant to oral diuretic therapy. The dose of furosemide ranged from 40 to $140 \mathrm{mg}$ given by SC bolus $(\mathrm{n}=5)$ or continuous infusion $(n=3) .{ }^{5}$ In a retrospective analysis of 43 episodes of decompensation in 32 advanced heart failure patients treated in hospital, community or hospice settings by a palliative care-heart failure collaborative, the continuous SC infusion of a dose of furosemide empirically based on their last daily oral dose and uptitrated according to response (dose range 40-250 mg daily) was demonstrably effective in relieving congestion, in facilitating early hospital discharge or in the prevention of admission in 26/28 episodes where this was the primary goal. ${ }^{6}$ Although the objective confirmation of a reduction in fluid overload was impractical in a subset of 15 hospice patients treated in the dying phase, none developed terminal dyspnoea.

\section{CLINICAL EXPERTISE}

Despite the relative paucity of published evidence as described above, there has been a widespread if variable adoption of the use of SC furosemide, reported in Switzerland, Spain, the UK and the USA. ${ }^{78}$ This practice has been endorsed in the palliative care drug formulary (palliativedrugs.com), and a series of protocols have been formulated for the symptomatic care of heart failure patients at the end of life. While it is accepted practice to implement novel off-license use of medication within a clinical protocol, there is a risk that this lends authority to such an approach. Prescribers may then assume this mode of furosemide administration is consistently effective, and fail to prescribe in the context of ongoing systematic assessment of the benefits and risks of such clinical management.

\section{PATIENT VALUES}

The proposal that maintenance of patient values be fundamental to evidence based medicine is consistent with a patient-centred approach to advanced heart failure care. Patient perspectives on improving current management have highlighted the importance of open sensitive dialogue in establishing a mutually agreed bespoke care plan to address their individual needs and coordination of care across all healthcare settings with defined responsibilities for informal and professional carers. There should also be regular review of goals of care along the disease trajectory to facilitate optimisation of treatment towards maintaining the quality of their remaining life. ${ }^{9}$ As evident in the study from Scarborough, 
the audited administration of SC furosemide would seem to provide a useful, well-tolerated means to relieve symptoms of congestion, while allowing patients to be cared for and die in their place of choice. ${ }^{6}$ However, it is important that this approach is discussed with the patient if possible so that the requirements of informed consent, patient choice and joint patient-clinician decision making are upheld.

Synthesis of the available data and observation of evolving clinical practice suggests that the current use of SC furosemide in the end of life care of advanced heart failure is only at the threshold of compliance with the three prima facie principles required of the definition of evidence based medicine. There are no randomised controlled trials in the treatment population, and the observational data is derived from small patient cohorts. Thus, at present, the use of SC furosemide must be regarded as largely intuitive. Given that some have already espoused this form of treatment, the genie may be out of the bottle, but questions remain regarding efficacy, effectiveness and safety. This element of heart failure care is no less worthy of rigorous systematic enquiry than the other forms of treatment offered earlier in the course of the illness. The burden of proof of this potentially beneficial therapy lies with us as clinicians and palliative medicine must not baulk from the requirement to develop a research base justifying its use. ${ }^{10}$ This evidence gap provides opportunities for study, and research themes might include (1) identifying the clinical subsets likely to respond; (2) elaboration of an effective dosing regimen and the logistics of drug administration; and (3) assessing the benefits and burdens of such treatment. This may well be a practical cost-effective means of providing parenteral loop diuretic therapy appropriate to a relatively frail advanced heart failure population across a variety of care settings, but we need to test that hypothesis.

\section{Competing interests None.}

Provenance and peer review Not commissioned; externally peer reviewed.

Received 9 August 2011

Accepted 9 November 2011

BMJ Supportive \& Palliative Care 2012;2:5-6.

doi:10.1136/bmjspcare-2011-000119

\section{REFERENCES}

1. Jaarsma T, Beattie JM, Ryder M, et al. Palliative care in heart failure: a position statement from the palliative care workshop of the Heart Failure Association of the European Society of Cardiology. Eur J Heart Fail 2009;11:433-43.

2. The use of drugs beyond licence in palliative care and pain management. A position statement prepared on behalf of the Association for Palliative Medicine and the British Pain Society. November 2005. http://www.britishpainsociety.org/book usingdrugs_main.pdf (accessed on 4 August 2011).

3. Sackett DL, Straus SE, Richardson WS, et al. Evidence-based Medicine: How to Practice and Teach EBM. Edinburgh: Churchill Livingstone 2000:3

4. Verma AK, da Silva JH, Kuhl DR. Diuretic effects of subcutaneous furosemide in human volunteers: a randomized pilot study. Ann Pharmacother 2004;38:544-9.

5. Goenaga MA, Millet M, Sánchez E, et al. Subcutaneous furosemide. Ann Pharmacother 2004:38:1751.

6. Zacharias H, Raw J, Nunn A, et al. Is there a role for subcutaneous furosemide in the community and hospice management of end-stage heart failure? Palliat Med 2011;25:658-63.

7. Herndon CM, Fike DS. Continuous subcutaneous infusion practices of United States hospices. J Pain Symptom Manage 2001;22:1027-34.

8. Fonzo-Christe C, Vukasovic C, Wasilewski-Rasca $A F$, et al. Subcutaneous administration of drugs in the elderly: survey of practice and systematic literature review. Palliat Med 2005;19:208-19.

9. Low J, Pattenden J, Candy B, et al. Palliative care in advanced heart failure: an international review of the perspectives of recipients and health professionals on care provision. J Card Fail 2011;17:231-52.

10. Currow DC. Why don't we do more rigorous clinical research so that we can stop experimenting on patients? J Palliat Med 2010;13:636-7. 